Europhysics Letters

PREPRINT

\title{
A promising method for the measurement of the local ac- celeration of gravity using Bloch oscillations of ultracold atoms in a vertical standing wave
}

\author{
Pierre Cladé ${ }^{1}$, Saïda Guellati-Khélifa ${ }^{2}$, Catherine Schwob $^{1}$, François Nez ${ }^{1}$, \\ LuCile Julien ${ }^{1}$ AND François Biraben ${ }^{1}$ \\ 1 Laboratoire Kastler Brossel, École Normale Supérieure, CNRS, UPMC, 4 place Jussieu, \\ 75252 Paris Cedex 05, France \\ 2 CNAM-INM, Conservatoire National des Arts et Métiers, 292 rue Saint Martin, \\ 75141 Paris Cedex 03, France
}

PACS. 32.80.Pj - Optical cooling of atoms; trapping.

PACS. 42.50.vk - Mechanical effects of light on atoms, molecules, electrons, and ions.

PACS. 04.80.-y - Experimental studies of gravity.

\begin{abstract}
An obvious determination of the acceleration of gravity $g$ can be deduced from the measurement of the velocity of falling atoms using a $\pi-\pi$ pulses sequence of stimulated Raman transitions. By using a vertical standing wave to hold atoms against gravity, we expect to improve the relative accuracy by increasing the upholding time in the gravity field and to minimize the systematic errors induced by inhomogeneous fields, owing to the very small spatial amplitude of the atomic center-of-mass wavepacket periodic motion. We also propose to use such an experimental setup nearby a Watt balance. By exploiting the $g / h$ ( $h$ is the Planck constant) dependence of the Bloch frequency, this should provide a way to link a macroscopic mass to an atomic mass.
\end{abstract}

Introduction. - The dynamics of an atomic wave packet in a periodic potential under the influence of a static force has been extensively analyzed using different physical approaches : in terms of Wannier-Stark resonance states [1], Bloch oscillations [2] or macroscopic quantum interferences induced by tunnelling due to the external acceleration [3](for review see [4]). An interesting configuration occurs when the external force is induced by the acceleration of gravity. In this case the Bloch frequency is equal to $\nu_{B}=\frac{m g \lambda}{2 h}$ and depends only on the local acceleration of the gravity $g$, the wavelength of the light $\lambda$ and some fundamental constants. This frequency is typically in the range $100 \mathrm{~Hz}-2000 \mathrm{~Hz}$, and its measurement allows the determination of $g$. Previous experiments have already been realized using the dynamics of BEC $[3,5]$ or degenerated Fermi gas [5] in vertical 1-D optical lattice. Kasevich's group has observed the interference between macroscopic quantum states of BEC atoms confined in a vertical array of optical traps. This interference arises from the tunneling induced by the acceleration of gravity and appears as a train of falling atomic pulses. The acceleration of the gravity $g$ was determined by measuring the spatial period of the pulses train. In Ingusio's group [5] the Bloch period is straightforwardly deduced from the evolution of the momentum

(c) EDP Sciences 
in the trap by adiabatically releasing the cloud from the lattice. In both experiments the detection is performed by imaging the falling atomic cloud using absorption imaging techniques and the uncertainty on the $g$ determination did not exceed $10^{-4}$ dominated by the imaging system.

Our experimental approach is based on the precise determination of the velocity distribution of atoms along the vertical axis using Doppler-sensitive Raman transitions. An obvious determination of the acceleration of the gravity is possible by measuring the atomic velocity variation after a given falling time $T$. This is performed by applying a $\pi-\pi$ pulses sequence with a spacing time $T$ : the first pulse defines an initial velocity by selecting a narrow velocity class from an ultracold atomic sample. Atoms are then in a well defined internal state. The second pulse measures the final velocity distribution of the atoms after the fall by transferring a resonant velocity slice to another internal state. This so-called velocity sensor allows us to locate the center of the velocity distribution with high accuracy and is now limited by the experimental setup platform's vibrations [6]. We could substantially improve the relative uncertainty on the measurement of $g$ by increasing the falling time $T$, but this parameter is swiftly limited by the dimension of the vacuum chamber. In our experiment we suggest to hold the atoms against gravity by applying between the two Raman pulses a far resonant standing wave, during an interrogation time $T_{\text {Bloch }}$ (fig. 1 a). In the non dissipative case, atoms fall until they absorb a photon from the upward wave and emit a stimulated photon in the downward wave. The atoms make a succession of $\Lambda$ transitions (fig. 1 b) inducing a momentum exchange of $2 \hbar k$ ( $k$ is a wave vector of the standing wave) with the cycling frequency $\nu_{B}=\frac{m g \lambda}{2 h}$. This evolution is equivalent to the dynamics of the Bloch oscillations [7]. In a previous work [8] we have measured a transfer efficiency of $99.5 \%$ per cycle. This result promises a large number of Bloch oscillations. Another particular interest of our method is the small spatial amplitude of the atomic wavepacket motion, during the oscillations $\left({ }^{1}\right)$, therefore the selection (first Raman pulse) and measurement (second Raman pulse) are done in a small volume allowing a better control of systematic effects arising from inhomogeneous fields. In this letter we investigate the possibility to make a high precise measurement of the acceleration of gravity $g$ using Bloch oscillations of cold atoms in a vertical standing wave. Such accurate measurements have important repercussions on geophysical applications including earthquake predictions, locating oil and studies of the global warming. We first describe our measurement method, then we present a preliminary measurement of $g$, and finally we discuss the signal losses observed when we increase the number of Bloch oscillations.

Experimental Set-up. - The main experimental apparatus has already been described in reference [8]. Briefly, ${ }^{87} R b$ atoms are captured, from a background vapor, in a $\sigma^{+}-\sigma^{-}$ configuration magneto-optical trap (MOT). The trapping magnetic field is switched off and the atoms are cooled to about $3 \mu \mathrm{K}$ in an optical molasses. After the cooling process, we apply a bias field of $\sim 100 \mathrm{mG}$. The atoms are then optically pumped into the $F=2, m_{F}=0$ ground state. The determination of the velocity distribution is performed using a $\pi-\pi$ pulses sequence of two vertical counter-propagating laser beams (Raman beams): the first pulse with a fixed frequency $\nu_{s e l}$, transfers atoms from $5 S_{1 / 2},\left|F=2, m_{F}=0\right\rangle$ state to $5 S_{1 / 2}$, $\left|F=1, m_{F}=0\right\rangle$ state, into a velocity class of about $v_{r} / 15$ centered around $\left(\lambda \nu_{\text {select }} / 2\right)-v_{r}$ where $\lambda$ is the laser wavelength and $v_{r}$ is the recoil velocity. To push away the atoms remaining in the ground state $\mathrm{F}=2$, we apply after the first $\pi$-pulse, a laser beam resonant with the $5 S_{1 / 2}(F=2)$ to $5 P_{1 / 2}(F=3)$ cycling transition. Atoms in the state $\mathrm{F}=1$ fall under the

$\left({ }^{1}\right)$ The amplitude of the oscillation of atomic wavepacket is given by $\Delta z=\Delta_{n} / 2|F|$, where $\Delta_{n}$ is the energy width of the nth band [2]. In our experiment only the fundamental band is considered $\Delta_{0}<E_{r}, F=m g$ Then $\Delta z<1 \mu \mathrm{m}$ which is very small compared to the size of the atomic cloud $(1 \mathrm{~mm})$. 


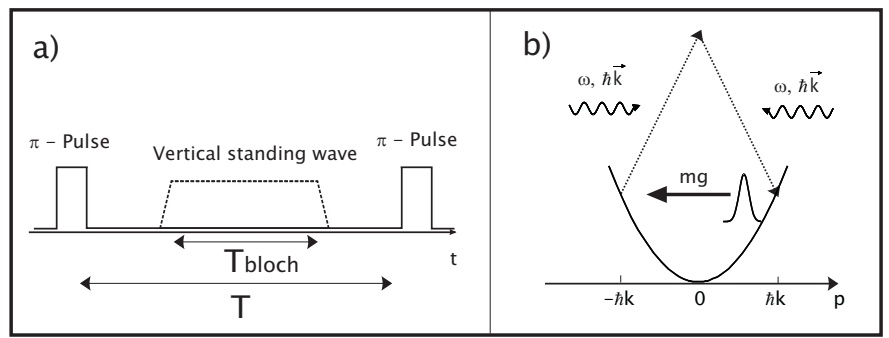

Fig. 1 - a). Experimental pulses sequence. b) A narrow velocity class is selected by the first Raman pulse. When the vertical standing wave is switched on, atoms fall until they are resonant with the $\Lambda$ transition: they absorb a photon from the upward wave and emit a stimulated photon in the downward wave. This induces a momentum exchange of $2 \hbar k$.

acceleration of gravity during $T$. We then perform the final velocity measurement using the second Raman $\pi$-pulse, whose frequency is $\nu_{\text {meas }}$. The population transfer from the hyperfine state $F=1$ to the hyperfine state $F=2$ due to the second Raman pulse is maximal when $2 \pi\left(\nu_{\text {sel }}-\nu_{\text {meas }}\right)=g \times T \times\left\|\left(\mathbf{k}_{\mathbf{1}}-\mathbf{k}_{\mathbf{2}}\right)\right\|$, where $\mathbf{k}_{\mathbf{1}}, \mathbf{k}_{\mathbf{2}}$ are the wave vectors of the Raman beams. The populations $(F=1$ and $F=2)$ are measured separately by using the one-dimensional time of flight technique developed for atomic clocks and depicted in [9]. To plot the final velocity distribution we repeat this procedure by scanning the Raman beam frequency $\nu_{\text {meas }}$ of the second pulse.

The two Raman beams are generated using two diode lasers injected by two extendedcavity diode lasers (ECLs). To drive the velocity-sensitive Raman transition, the frequency difference of the master lasers must be precisely resonant with ${ }^{87} R b$ ground-state hyperfine transition $(\sim 6.8 \mathrm{GHz})$. The frequency of one ECL is stabilized on a high stable Zerodur Fabry-Perot (ZFP) cavity. The second ECL is then phase-locked to the other using the beat note technique (see fig. 2 b). The very stable RF source is performed by mixing the $62 \mathrm{th}$ harmonic of a 100 MHZ quartz oscillator with different digital synthesizers (SRS DS345). They are used to tune finely the frequency of the RF source. A YIG oscillator is phase-locked on to the central line of the source in order to reject completely the residual sidebands of the different mixings. A multiplexer switches between two synthesizers to generate the frequency offset for the velocity selection or measurement. The frequency of a third synthesizer is linearly swept during the selection and the measurement pulses to compensate the Doppler shift during the fall of the atoms (fig. 2a). The time interval between the two Raman pulses is precisely defined by the delay between the triggering signal of the two frequency sweeps. Each laser beam passes through an acousto-optic modulator $(\sim 80 \mathrm{MHz})$ for timing (switch on and off) and intensity control. The two beams have linear orthogonal polarizations and are coupled into the same polarization maintaining optical fiber. The pair of Raman beams is sent through the vacuum cell. The counter propagating configuration is achieved using a polarizing beam-splitter cube and an horizontal retroreflection mirror placed above the exit window of the cell. The standing wave used to create the 1-D optical lattice is generated by a Ti:Sapphire laser, whose frequency is stabilized on the same highly stable ZFP cavity. This laser beam is splitted in two parts. To perform the timing sequence, each one passes through an acousto-optic modulator to control its intensity and frequency. The beams are detuned by $260 \mathrm{GHz}$ from the $5 S_{1 / 2}-5 P_{3 / 2}$ resonance line to avoid spontaneous emission. With these laser parameters, the optical potential depth $U_{0}$ equals to $2.7 E_{R}\left(E_{R}=\frac{\hbar^{2} k^{2}}{2 m}\right.$ is the recoil energy). For this value, when the external acceleration is due to gravity, the transfer of atoms 


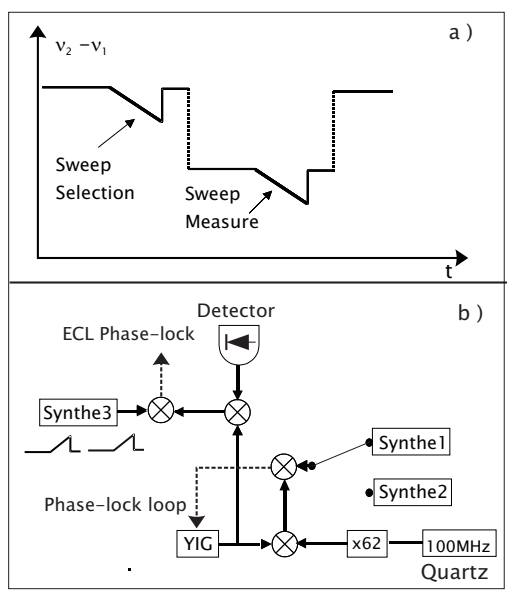

Fig. 2

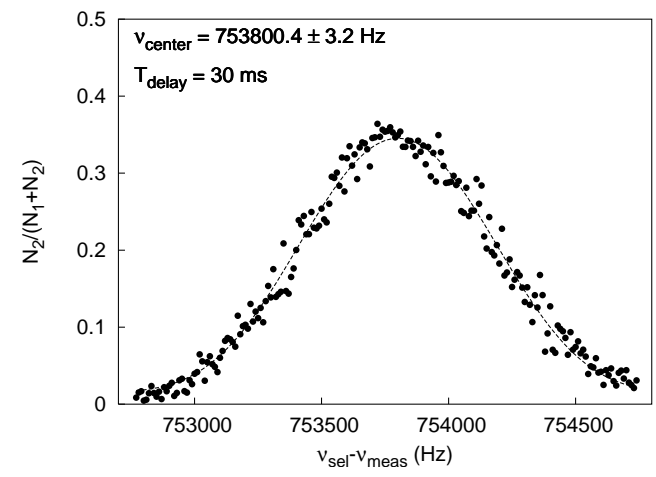

Fig. 3

Fig. 2 - a). Temporal variation of the frequency difference of the two Raman beams. b). The synthe1 and the synthe 2 allow us to switch between the selection and a measurement steps. To compensate the Doppler shift during the falling of the atoms the frequency of the synthes 3 is swept linearly during the Raman pulses.

Fig. 3 - The final distribution velocity of atoms after a free fall of $30 \mathrm{~ms}$. The center of the velocity distribution is located with an uncertainty of $3.2 \mathrm{~Hz}$, corresponding to a relative uncertainty of $4 \times 10^{-6}$ on the measurement of the acceleration of the gravity .

to the higher bands remains insignificant for several periods of Bloch oscillations.

Results. - In a first experiment, we determine $g$ by measuring the atomic velocity variation after the free fall of atoms during $30 \mathrm{~ms}$, using the $\pi-\pi$ Raman pulses sequence described previously. The typical final velocity distribution is shown in (fig. 33). The center of this distribution is located with an uncertainty of $3.2 \mathrm{~Hz}$ (corresponding to $v_{r} / 5000$ ) in an average time of $20 \mathrm{mn}$, allowing a measurement of $g$ with a relative uncertainty of $4 \times 10^{-6}$. This uncertainty is limited by many systematic errors. These errors may occur due to the vibration noise of the retroreflecting mirror [6], the fluctuation of the number of detected atoms and the atomic motion between the two pulses (effect of inhomogeneous fields).

In a second experiment, we apply between the two $\pi$-pulses a standing wave during an interrogation time $T_{\text {Bloch }}$. We then study the evolution of the final momentum distribution by changing $T_{\text {Bloch }}$. Before analyzing the experimental results, we briefly recall the relevant results of Bloch's theory. The energy spectrum of the particle presents a band structure (indexed by $n$ ) arising from the periodicity of the potential (optical lattice with period $d=$ $\lambda / 2$ ). The corresponding eigenenergies $E_{n}(q)$ and the eigenstates $|n, q\rangle$ (Bloch states) are periodic functions of the continuous quasi-momentum $q$, with a period $2 k=2 \pi / d$. The quasimomentum $\mathrm{q}$ is conventionally restricted to the first Brillouin zone $]-\pi / d, \pi / d]$. If we apply a constant force $F$, sufficiently weak in order to avoid interband transitions, a given Bloch state $|n, q(0)\rangle$ evolves (up to a phase factor) into the state $|n, q(t)\rangle$ according to

$$
q(t)=q(0)+2 k \frac{t}{\tau_{B}} \quad(\bmod 2 \pi / d)
$$

When the atoms are only submitted to the gravity force, the Bloch period $\tau_{B}$ is given 
by $\frac{2 h}{m g \lambda}$. This period corresponds to the time required for the quasi-momentum to scan a full Brillouin zone. In our experiment, first we prepare Bloch states around $q=0$ (in lattice frame) at the bottom of the fundamental energy band $(n=0)$ by turning on adiabatically the standing wave (rise time of $300 \mu \mathrm{s}$ ): this avoids a transfer of population into the higher energy band. We point out that just before turning on the Bloch potential, selected atoms reach a mean velocity of about $10 v_{r}$. In order to compensate this velocity drift, the upward beam's frequency is shifted by $\sim 150 \mathrm{kHz}$ (in the laboratory frame the standing wave is then moving with a constant velocity of about $10 v_{r}$ ). To use a pure standing wave, we should launch atoms in ballistic atomic-fountain trajectories either from a moving molasses [10] or with Bloch oscillations [8], and turn on the Bloch potential when they reach their summit. After time $T_{B}$ we suddenly switch off the optical potential and we measure the final momentum distribution in the first Brillouin zone. In (fig. 4 a) we report the center of the measured peak as a function of the holding time $T_{B}$. The observed sawtooth shape is the signature of Bloch oscillations (eq. (1). We observe more than 60 Bloch periods corresponding to the longest lived Bloch oscillator observed in bosonic systems. To determine the Bloch period $\tau_{B}$, we measure the time interval between the centers of the two extreme slopes of sawtooth. We extract the value of $\tau_{B}$ by dividing this time interval by the number of periods. This measurement leads to a determination of the local acceleration of gravity with a relative uncertainty of $1.110^{-6}$ as $\frac{h}{m}$ ratio [11] and the wavelength $\lambda$ are known with a better accuracy. The linear fit of the experimental data in (fig. 目a) is performed by fixing the value of the recoil velocity. In fig. 4. b we present the residuals of the fit; they increase when we move away from the center of the Brillouin zone. That reveals that there is a difference between the quasi-momentum and the measured momentum of about $10^{-3} \times \hbar k$. The momentum spectrum $\Psi_{0, q}(p)$ can be calculated by projecting the Bloch state $|0, q\rangle$ onto plane-wave components $|p\rangle$ (measurement basis). Using analytic properties of the Bloch wavefunctions one obtains

$$
\Psi_{0, q}(p)=\sqrt{\frac{2 \pi}{d}} \tilde{\Phi}_{0}(p) \times \sum_{l} \delta(p-q-2 \pi l / d), \quad l \in Z
$$

where $\tilde{\Phi}_{0}(p)$ is the Fourier transform of the Wannier function of the fundamental energy band [12]: the momentum spectrum is composed of peaks separated by the reciprocal-lattice vector, $2 k=2 \pi / d$, with an amplitude given by the envelope function $\tilde{\Phi}_{0}(p)$. This function is not constant along the width of the selected velocity class. Thus the center of the measured momentum distribution is shifted with respect to the quasi-momentum $q(t)$ (eq 1) by a factor depending on the momentum spread. The data analysis allowing the determination of the Bloch period is performed in order to reduce substantially the systematic error induced by this effect.

When we increase the interrogation time of the standing wave up to $100 \mathrm{~ms}$, the signal is significantly degraded (loss rate becomes larger than 50\%). In order to understand the origin of this losses, we have measured, for a given laser intensity, the losses per oscillation versus the detuning $\Delta$, relative to $F=2 \rightarrow F=3$ transition (fig. 5 ). These losses are obtained by comparing the number of atoms measured after $N$ oscillations to those measured after $N=10$ oscillations. The choice of the parameter $\Delta$ allows us to estimate the effects of the spontaneous photon scattering and the interband transitions which depend both on $\Delta$ (for interband transition see [7]). We observe that the losses exceed 3\%; they are more important than the $0.5 \%$ rate losses measured using an accelerated standing wave [8]. This results from the slowness of the Bloch oscillations, since in a vertical standing wave the Bloch period is $\sim 1.2 \mathrm{~ms}$, when in reference [8] the period was only $\sim 0.1 \mathrm{~ms}$.

For the experimental values of the standing wave parameters, the losses induced by the 

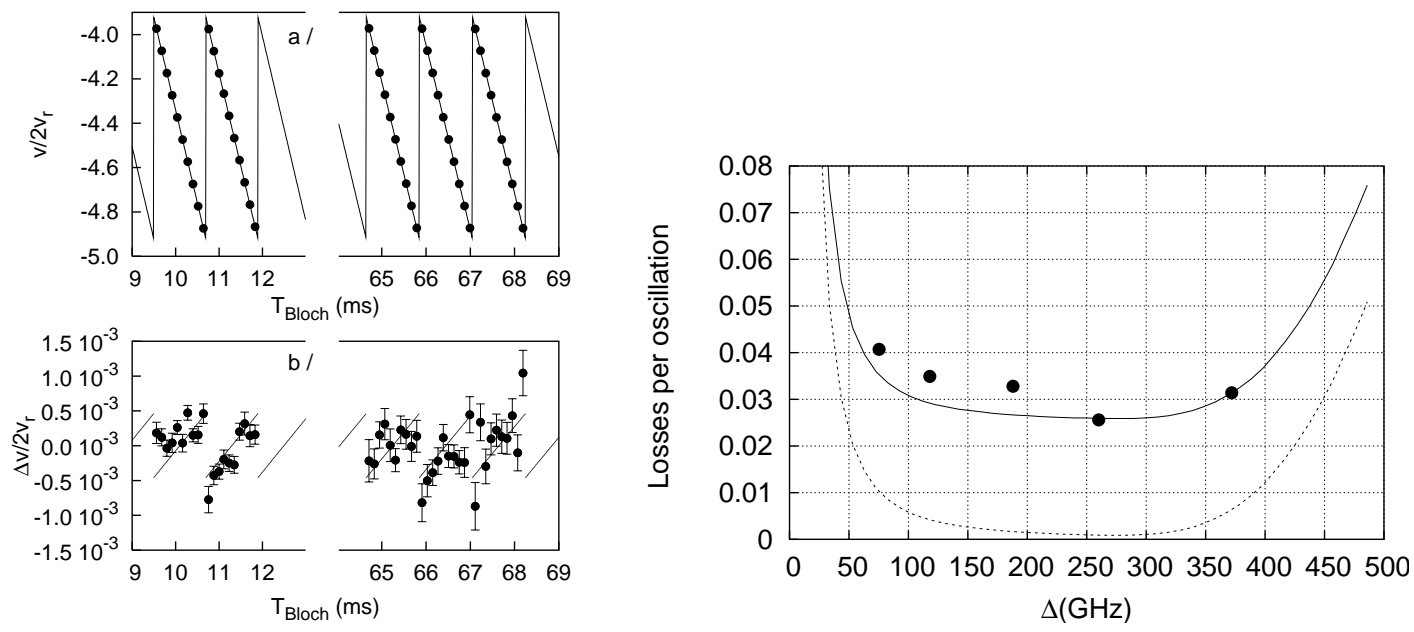

Fig. 4

Fig. 5

Fig. 4 - a. The center of the final velocity distribution versus the duration of the standing wave. The dot represent the experimental data and the line the least-square fit performed by fixing the recoil velocity. b) The residuals of the fit.

Fig. 5 - The losses per oscillation versus detuning $\Delta$. These losses are obtained by comparing the number of atoms measured after $N$ oscillations to those measured after $N=10$ oscillations. The relevant advantage of this presentation is to take into account only the losses during the Bloch oscillations process. Dot: the experimental data. The theory: (Dotted line). Solid line: theory including the interparticle collisions.

spontaneous emission and the interband transitions in the weak binding limit do not match with the experimental data (dotted line in (fig. 5). Performing a least-square fit based on this model and including collisions with the residual $\mathrm{Rb}$ vapor, we extract a characteristic time constant of the damping due to the collisions of about $70 \mathrm{~ms}$. This value corresponds to the lifetime of the molasses for the residual vapor pressure in the cell. Therefore we think, that the number of Bloch oscillations, in our experiment could be increased by reducing the pressure in the vacuum chamber.

Conclusion and prospects. - We have described an experimental method to measure the vertical velocity distribution of atoms by using a $\pi-\pi$ pulses sequence of Doppler-sensitive Raman transitions. We have performed a preliminary determination of the local acceleration of gravity with a relative accuracy of $10^{-6}$ by measuring the Bloch period. We have also demonstrated that the number of Bloch oscillations is not yet limited by either the interband transitions or the spontaneous emission, but only by collisions with the background atomic vapor. To overcome this limit, we are now building a new ultrahigh vacuum chamber where the magneto-optical trap will be loaded by an atomic slow beam. In order to improve the accuracy of the velocity measurement a vibration-isolation system is also in implementation. These improvements should allow us to take a better benefit of the Bloch oscillations.

An attractive possibility consists to replace the $\pi-\pi$ velocity measurement by a two $\pi / 2$ Ramsey-Bordé sequence. We obtain then a $\pi / 2-\pi / 2$-Bloch oscillation- $\pi / 2-\pi / 2$ atominterferometer. Comparing this scheme to the $\pi / 2-\pi-\pi / 2$ atom-interferometer used in gravimetry $[13,14]$, where the pulses spacing time is limited by the effects related to the 
spatial position, the advantage would be to increase the measurement time thanks to Bloch oscillations for a similar phase difference between the two paths. This way, we could reduce significantly the uncertainty of the gravity interferometric measurement. Finally, we suggest to use such experiment nearby a Watt balance site $[15,16]$. In the dynamics mode of the balance the relation which equates the mechanical power and the electrical power is given by $[16]$ :

$$
M g v=C F_{J} F_{J}^{\prime} h
$$

where $\mathrm{M}$ is the standard mass, $v$ is the velocity of the vertical moving coil, $h$ the Planck constant, $C$ represents a dimensionless constant and $F_{J}, F_{J}^{\prime}$ denote the frequencies applied to a Josephson device. Using the $g / h$ dependence of the Bloch period we obtain

$$
\frac{M}{m}=\frac{C F_{J} F_{J}^{\prime}}{2} \frac{\lambda}{\nu_{B}} \frac{1}{v}
$$

In conclusion, associating our Bloch oscillations experiment to a Watt Balance could be used to link a macroscopic mass to an atomic mass.

$$
* * *
$$

We thank A. Clairon and C. Salomon for valuable discussions. This experiment is supported in part by the Bureau National de Métrologie (Contrats 993009 and 033006) and by the Région Ile de France (Contrat SESAME E1220).

\section{REFERENCES}

[1] Wilkinson S.R, Bharucha C.F, Madison K.W, Qian Niu and Raizen M.G, Phys.Rev.lett, 76 (1996) 4512.

[2] Ben Dahan M., Peik E, Reichel J., Castin Y. and Salomon C., Phys. Rev. Lett., 76 (1996) 4508.

[3] Anderson B.P. and Kasevich M.A., Science, 282 (1998) 1686.

[4] Raizen M.G., Salomon C. and Niu .Q, Phys. Today, 50 (1997) 30.

[5] Roati G.,De Mirandes E., Ferlaino F., Ott H., Modungno G. and Inguscio M., Phys.Rev.lett, 92 (2004) 230402-1.

[6] Cladé P.,Guellati-khélifa S., Schwob C., Nez F., Julien F. and Biraben F., Eur. Phys. J. D, 33 (2005) 173.

[7] Peik E., Ben Dahan M., Bouchoule I., Castin Y. and Salomon C.,Phys. Rev. A, 55 (1997) 2989.

[8] Battesti. R, Cladé P., Guellati-Khélifa S., Schwob C., Grémaud B., Nez F., Julien L. and Biraben F.,Phys. Rev. Lett., 92 (2004) 253001-1.

[9] Clairon A., Laurent P., Santarelli G., Ghezali S., Lea N. and Bahoura M., IEeE Trans. Instrum. Meas., 44 (1995) 128.

[10] Clairon A., Salomon C., Guellati S. and Phillips W.D., Europhys.Lett, 16 (1991) 165.

[11] Mohr P. and Taylor B., Rev. Mod. Phys., 72 (2000) 351.

[12] Kohn. W, Phys. Rev., 115 (1959) 809.

[13] Peters A., Chung K.Y. and Chu S., Metrologia, 38 (2001) 25.

[14] McGuirk J.M., Foster G.T., Fixler J.B., Snadden M.J. and Kasevich M.,Phys. Rev. A, 65 (2002) 033608.

[15] Kibble. B.P, Atomic Mass and Fundamental Constants, edited by J.H. Sanders and A.H. Wapstra (Plenum, New York,1976), 5 (545).

[16] Eichenberger. A, Jeckelmann. B, and Richard. P., Metrologia, 40 (2003) 356-365. 